
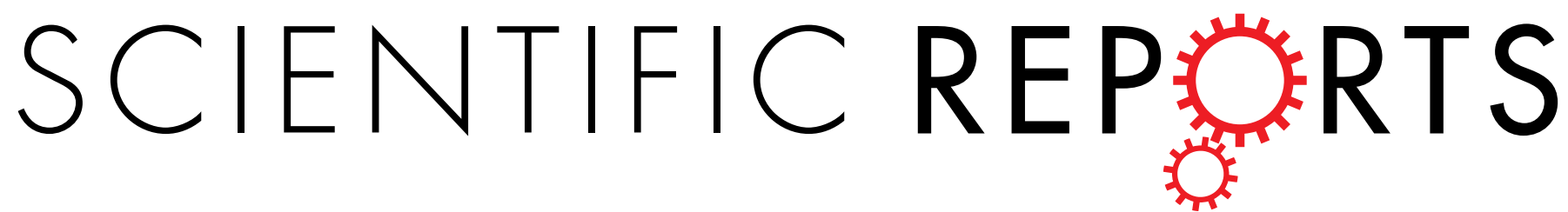

\title{
OPEN
}

Received: 02 March 2015

Accepted: 19 June 2015

Published: 20 July 2015

\section{Opportunistic pathogen Candida albicans elicits a temporal response in primary human mast cells}

José Pedro Lopes ${ }^{1,2,3, *}$, Marios Stylianou ${ }^{1,2,3, *}$, Gunnar Nilsson ${ }^{4} \&$ Constantin F. Urban ${ }^{1,2,3}$

Immunosuppressed patients are frequently afflicted with severe mycoses caused by opportunistic fungal pathogens. Besides being a commensal, colonizing predominantly skin and mucosal surfaces, Candida albicans is the most common human fungal pathogen. Mast cells are present in tissues prone to fungal colonization being expectedly among the first immune cells to get into contact with C. albicans. However, mast cell-fungus interaction remains a neglected area of study. Here we show that human mast cells mounted specific responses towards $C$. albicans. Collectively, mast cell responses included the launch of initial, intermediate and late phase components determined by the secretion of granular proteins and cytokines. Initially mast cells reduced fungal viability and occasionally internalized yeasts. C. albicans could evade ingestion by intracellular growth leading to cellular death. Furthermore, secreted factors in the supernatants of infected cells recruited neutrophils, but not monocytes. Late stages were marked by the release of cytokines that are known to be anti-inflammatory suggesting a modulation of initial responses. C. albicans-infected mast cells formed extracellular DNA traps, which ensnared but did not kill the fungus. Our results suggest that mast cells serve as tissue sentinels modulating antifungal immune responses during $C$. albicans infection. Consequently, these findings open new doors for understanding fungal pathogenicity.

Severe mycoses are rising in modern health care, mainly due to the use of catheters and immunosuppressive treatments ${ }^{1}$. The most prevalent fungal pathogen ${ }^{2}$, Candida albicans is also part of the human commensal flora. C. albicans commensally colonizes the gastrointestinal, urogenital, oral-nasal cavity and skin. When host immunity is suppressed, C. albicans can disseminate to non-commensal niches, resulting in hazardous colonization and invasive disease. C.albicans-associated mycoses have an annual prevalence of 300000 with an associated mortality up to 55\% in European intensive care units ${ }^{3,4}$. Due to their distribution in tissues facing external surfaces mast cells are among the first immune cells to get in contact with C. albicans.

Mast cells are tissue-dwelling cells derived from hematopoietic progenitors. These cells migrate from the blood to the skin, airways or the gastrointestinal tract where final differentiation is induced by surrounding structural cells. Mast cells are known for triggering hypersensitivity reactions at the body interfaces with external environments. They respond to stimuli by rapidly degranulating their cytoplasmic vesicles leading to selective and differential mobilization of granule contents into the milieu.

The role of mast cells in asthma and inflammatory disorders is an intensively active area of research, however, comparably little is known about the role of these cells in host defence ${ }^{5}$. Mast cells have been shown to participate in the killing of bacteria ${ }^{6-8}$, whereas their antifungal defence response is

${ }^{1}$ Department of Clinical Microbiology, Umeå University, Umeå, Sweden. ${ }^{2}$ Umeå Centre for Microbial Research (UCMR). ${ }^{3}$ The Laboratory for Molecular Infection Medicine Sweden (MIMS). ${ }^{4}$ Department of Medicine, Karolinska Institutet and Karolinska University Hospital, Stockholm, Sweden. ${ }^{*}$ These authors contributed equally to this work. Correspondence and requests for materials should be addressed to C.F.U. (email: constantin.urban@umu.se) 
virtually unexplored ${ }^{9-12}$. Interestingly, mast cells express molecules, for instance inflammatory cytokines, myeloid-attracting chemokines, and pattern recognition receptors that were demonstrated to be involved in antifungal responses in other cells. Toll like receptors (TLRs), such as TLR2 and TLR4 are in addition to mediate responses against bacteria ${ }^{13-15}$ well-established contributors for detection and clearance of fungi ${ }^{16,17}$. Activation of C-type lectin receptors, such as dectin 1, by fungal components also results in mast cell activation ${ }^{18,19}$. This suggests a possible involvement of mast cells in antifungal immunity. Specific ligands for such receptors can activate mast cells causing release of reactive oxygen species ${ }^{20}$ and different vasoactive mediators, for instance histamine, prostaglandins, leukotrienes, and tryptase, which are injurious to the microbes ${ }^{21}$. While most of these mediators promote inflammation, they are in addition responsible to recruit other immune cells ${ }^{22,23}$.

We chose $C$. albicans to study fungal-mast cell interactions, since $C$. albicans is a commensal and a frequent human pathogen. This dual role enables a more detailed understanding of fungal pathogenicity, innate immune response and immune tolerance. We found that human mast cells have a versatile and timed response upon fungal encounter. Mast cells first degranulated $\beta$-hexosaminidase and were able to transiently reduce $30 \%$ of $C$. albicans viability up to $3 \mathrm{~h}$ post infection. In intermediate responses mast cells released pro-inflammatory cytokines, such as interleukin-8 (IL-8) and supernatants of C. albicans-infected mast cells were chemoattractive to neutrophils. In late responses mast cells secreted IL-16 and anti-inflammatory IL-1ra and released mast cell extracellular traps (MCETs) that ensnared, but probably did not kill $C$. albicans. In addition, the fungus could cause mast cell death by different mechanisms.

Ultimately, our work contributes to the understanding of the role of mast cells in modulating the innate immune response against opportunistic pathogenic fungi.

\section{Results}

C. albicans induced rapid degranulation in mast cells. Mast cells contain large amounts of enzymes in their granules ${ }^{21}$, particularly proteases or lysosomal enzymes like $\beta$-hexosaminidase ${ }^{24}$. These enzymes are involved in inflammation onset ${ }^{25,26}$ and in defence against microbes ${ }^{27-29}$. Degranulation is therefore a putative mechanism mast cells may employ to respond to C. albicans infection. Therefore, we measured $\beta$-hexosaminidase, a routinely used marker for mast cell degranulation, during infection of mast cells with $C$. albicans. Indeed, mast cells degranulated and released $\beta$-hexosaminidase in response to $C$. albicans after $1 \mathrm{~h}$ of infection in a dose-dependent manner (Fig. 1A). This indicates that mast cells recognized the fungus and mounted an early and direct response.

Mast cells mounted a unique cytokine response upon $C$. albicans infection. To test mast cell immune modulatory responses we infected human mast cell line-1 (HMC-1) cells with C. albicans and subsequently analysed culture supernatants for presence of cytokines. We found 5 cytokines that were differentially released from mast cells in a time-dependent manner following infection with C. albicans. An early cytokine response ( $6 \mathrm{~h}$ post infection) involved release of IL-8, a strong neutrophil chemoattractant (Fig. 1B). Comparably, cord blood-derived mast cells released similar amounts of IL-8 upon $C$. albicans infection (Fig. S1).

Upon C. albicans stimulation, mast cells additionally secreted macrophage migration inhibitory factor (MIF), a pro-inflammatory, stress-response cytokine crucial for sustaining an inflammatory milieu (Fig. 1C) $)^{30}$. Interestingly, secretion of monocyte chemoattractant protein 1 (MCP-1), one of the key chemokines inducing migration and infiltration of monocytes/macrophages was not released (Fig. 1D). Mast cells therefore are likely to contribute to neutrophil, but not to macrophage recruitment upon C. albicans infection. At later time points ( 12 and $24 \mathrm{~h})$, the cytokine profile revealed the release of IL-16, a chemokine linked to chemoattraction of $\mathrm{CD}^{+}{ }^{+} \mathrm{T}$ lymphocytes ${ }^{31}$ (Fig. 1E). The pro-inflammatory cytokine response at early time points post infection seems to be counteracted by release of the anti-inflammatory cytokine IL-1ra at $24 \mathrm{~h}$ (Fig. 1F). Taken together, these data suggests that secretion of pro- and anti-inflammatory cytokines was a controlled process that was influenced by different stages of the infection.

Human neutrophils but not monocytes were chemoattracted towards $C$. albicans-infected mast cells. As some of the chemokines from our multiplex screening are relevant in host immune cell recruitment, we next tested the chemoattractive potential of supernatants from C. albicans-infected mast cells towards neutrophils and monocytes. Mast cells were infected for three time points and supernatants harvested. The chemotactic potential of the supernatants was tested using fluorescently labelled neutrophils in a transwell system (Fig. 2A). Chemoattractant fMLF was used as positive control ${ }^{32}$.

Notably we found, that $C$. albicans-infected supernatants induced migration of neutrophils similar to fMLF, whereas C. albicans alone or uninfected mast cells induced significantly lower neutrophil migration. Chemotaxis was significantly above controls with supernatants collected after $12 \mathrm{~h}$ or longer. The migration assay shows slightly delayed neutrophil chemotaxis compared to the cytokine release assay revealing increased IL-8 already after $6 \mathrm{~h}$ of infection (Fig. 1B). However, neutrophil migration might be influenced by other chemokines that were not analysed with the multiplex assay used. On the other hand analysis of monocyte chemotaxis corroborated the cytokine multiplex results. Monocyte-attractant chemokine MCP-1 was secreted by uninfected mast cells, however not induced upon C. albicans infection 

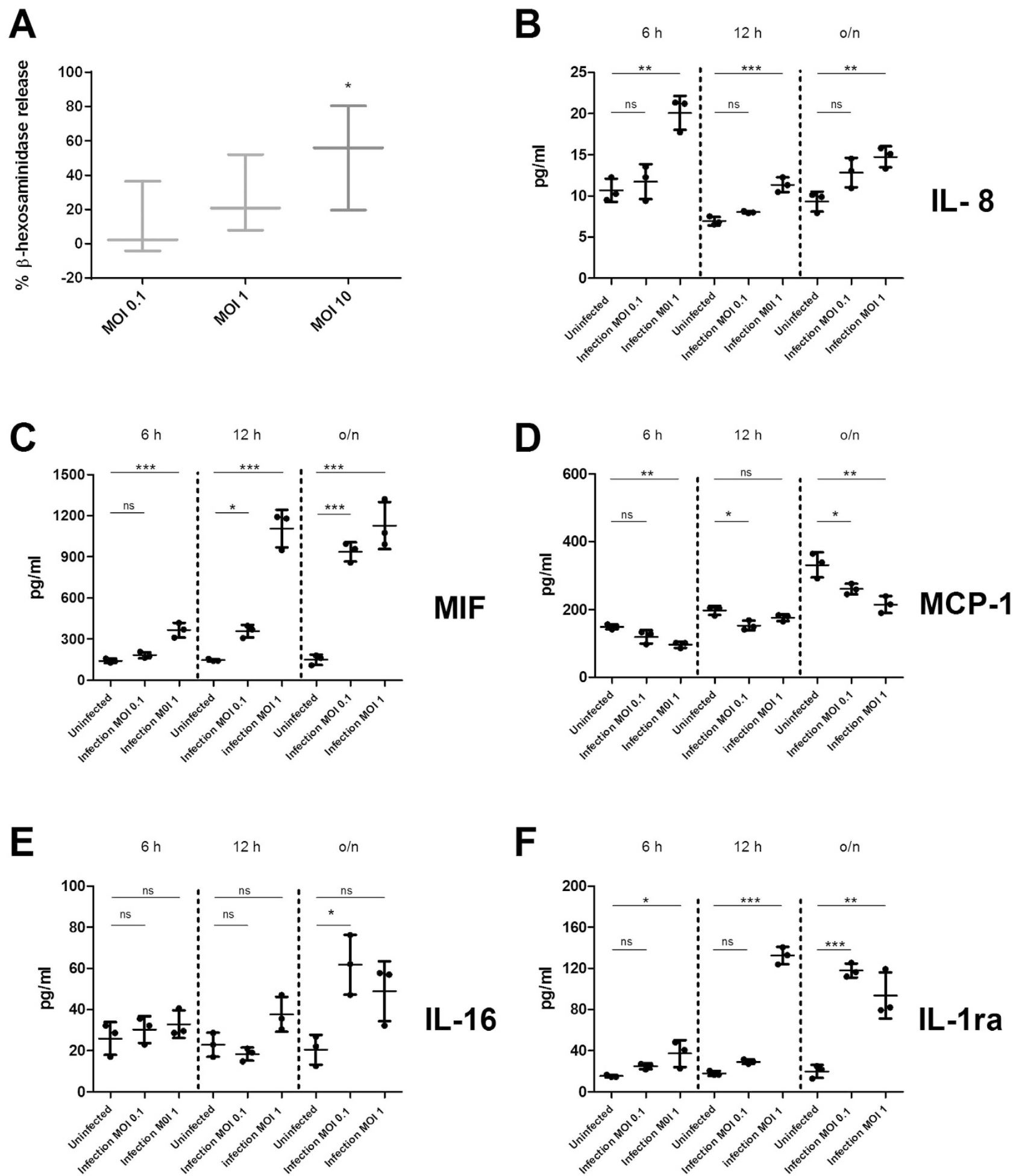

Figure 1. C. albicans induced mast cell degranulation and cytokine release in a MOI-dependent manner. (A) HMC-1 cells were infected with opsonized C. albicans yeasts (MOI 0.1, 1 and 10) for 1 hour, after which B-hexosaminidase release was measured from supernatants of infection. (B-F) Shown are 5 cytokines at 6,12 and $24 \mathrm{~h}$ post infection that were released differentially from different supernatants of mast cells infected with C. albicans (MOI 0.1 and 1) or of mast cells left uninfected. $ß$-hexosaminidase percentage release was defined by the amount of $\beta$-hexosaminidase release from infected cell divided by spontaneous $ß$-hexosaminidase release from uninfected cells ( $\%$ of $\beta$-hexosaminidase release/\% of $B$-hexosaminidase control). Significance for (A-F) was analysed by Tukey one-way ANOVA ${ }^{\star} \mathrm{P} \leq 0.05$. Data are presented as means of $\mathrm{n}=4$ (4) $\pm \mathrm{SD}$ (ß-hexosaminidase release assay) and $\mathrm{n}=3$ (3) $\pm \mathrm{SD}$ (Cytokine Multiplex). 

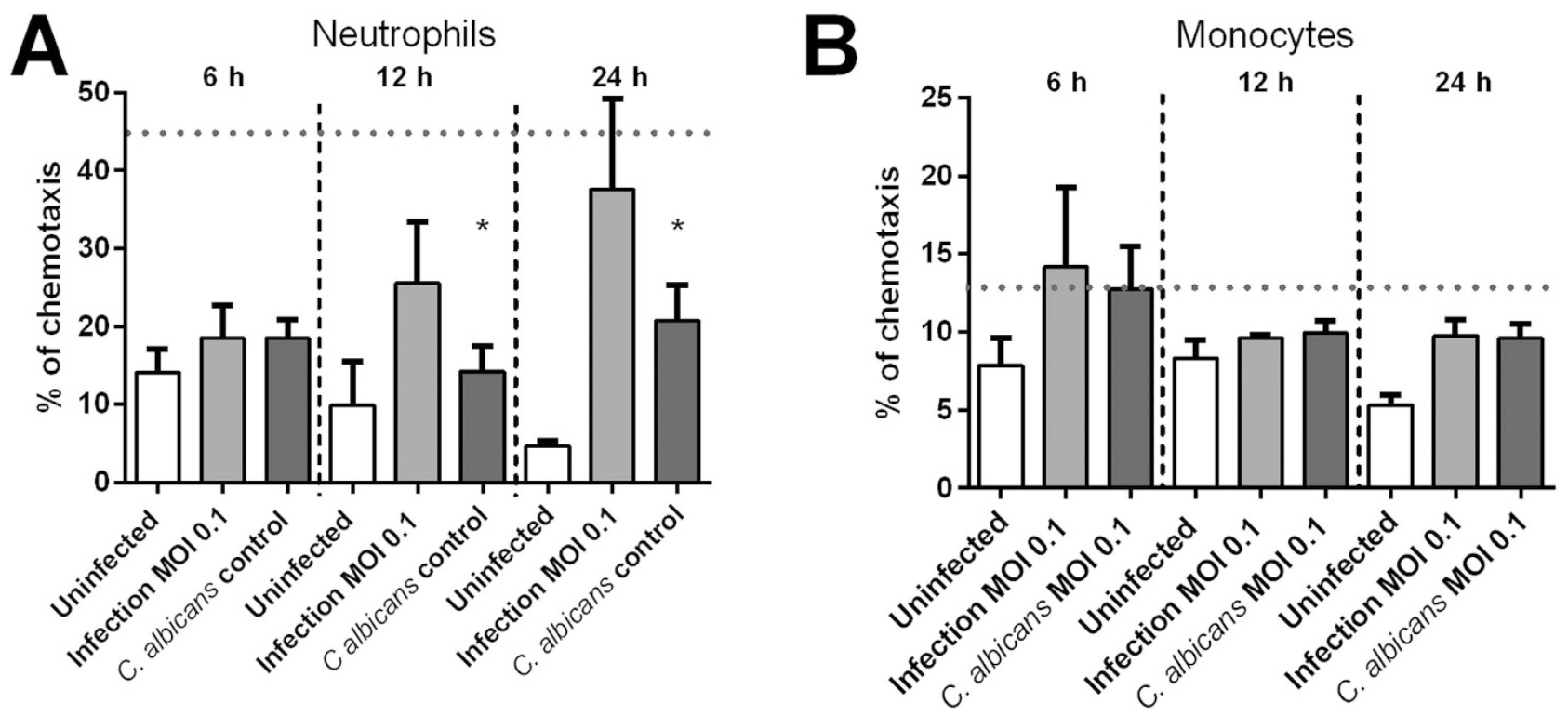

Figure 2. Neutrophils but not monocytes differentially migrated in response to supernatants from mast cells infected with $C$. albicans. Supernatants collected from C. albicans-infected mast cells (MOI 0.1) at 6h, $12 \mathrm{~h}$ and overnight infection were used as chemoattractants to neutrophils and monocytes in a transwell system. End-point cell migration was plotted per condition, per time as ratio of migrated cells using as $100 \%$ control cells added to the lower compartment without inserted transwell system. (A) Neutrophil migration is increased over time towards supernatants of infection but not to C. albicans and HMC-1 alone (controls). (B) Monocytes show no significant chemoattraction towards supernatants of infected mast cells. Variations between neutrophil or monocyte migration over time towards supernatants of infection and C. albicans control were analysed for statistical significance using a one-way ANOVA with Tukey post-test. As positive control for migration we used fMLP causing chemotaxis significantly above background of approximately $45 \%$ after $30 \mathrm{~min}$ for neutrophils and $13 \%$ after $90 \mathrm{~min}$ for monocytes. These values are indicated as a horizontal, dashed line in the graphs of the figure. Data are presented as means of $n=5(3) \pm S D$.

of mast cells (Fig. 1D). In contrast, monocyte-inhibitory MIF was increasingly released by infected mast cells over time (Fig. 1C).

Taken together, we confirmed our findings regarding mast cells cytokine-release following fungal infection by a functional migration assay revealing that mast cells secrete neutrophil chemoattractants.

Despite releasing extracellular traps mast cells only transiently control C. albicans viability. The release of extracellular DNA traps is part of the innate immune response to infection. Extracellular traps have been observed in mast cells in response to auto-inflammatory skin diseases and upon bacterial infection ${ }^{6,14,33}$. Here, we investigated the potential release of MCETs in response to fungi. After 6h, C. albicans-infected mast cells released MCETs composed of DNA and granular proteins ensnaring the fungus (Fig. 3A arrow, Movie S1), whereas these structures were absent in uninfected control (Fig. 3B). Primary mast cells similarly released extracellular traps upon C. albicans stimulation (Fig. $\mathrm{S} 2 \mathrm{~A}$, arrow). Quantification of MCETs in a blinded fashion revealed that extracellular trap formation increased over time being significantly different from uninfected controls, but rarely exceeded $5 \%$ of the total amount of cells (Fig. 4A). Infection with higher MOIs led to MCET formation (Fig. S2B, arrow) without further increase in number (data not shown).

To account for mast cell antifungal activity, we quantified ATP levels correlating with the presence of metabolically active fungal cells. Within $3 \mathrm{~h}$ of incubation C. albicans viability was reduced by $30 \%$. However, this antifungal effect was transient and declined after $6 \mathrm{~h}$, possibly due to fungal overgrowth.

In a similar assay, we tested the antifungal activity of MCETs by degrading their DNA backbone with DNase. The nuclease was added before infection of mast cells with $C$. albicans and not removed during the whole assay to ensure degradation of any emerging MCET. We did not observe a significant difference in fungal viability in the presence of DNase as compared to samples without DNase (Fig. 4B). We conclude that mast cells display moderate antifungal activity, which appears to be MCET-independent. The traps, nevertheless, ensnared C. albicans (Fig. 3A). 

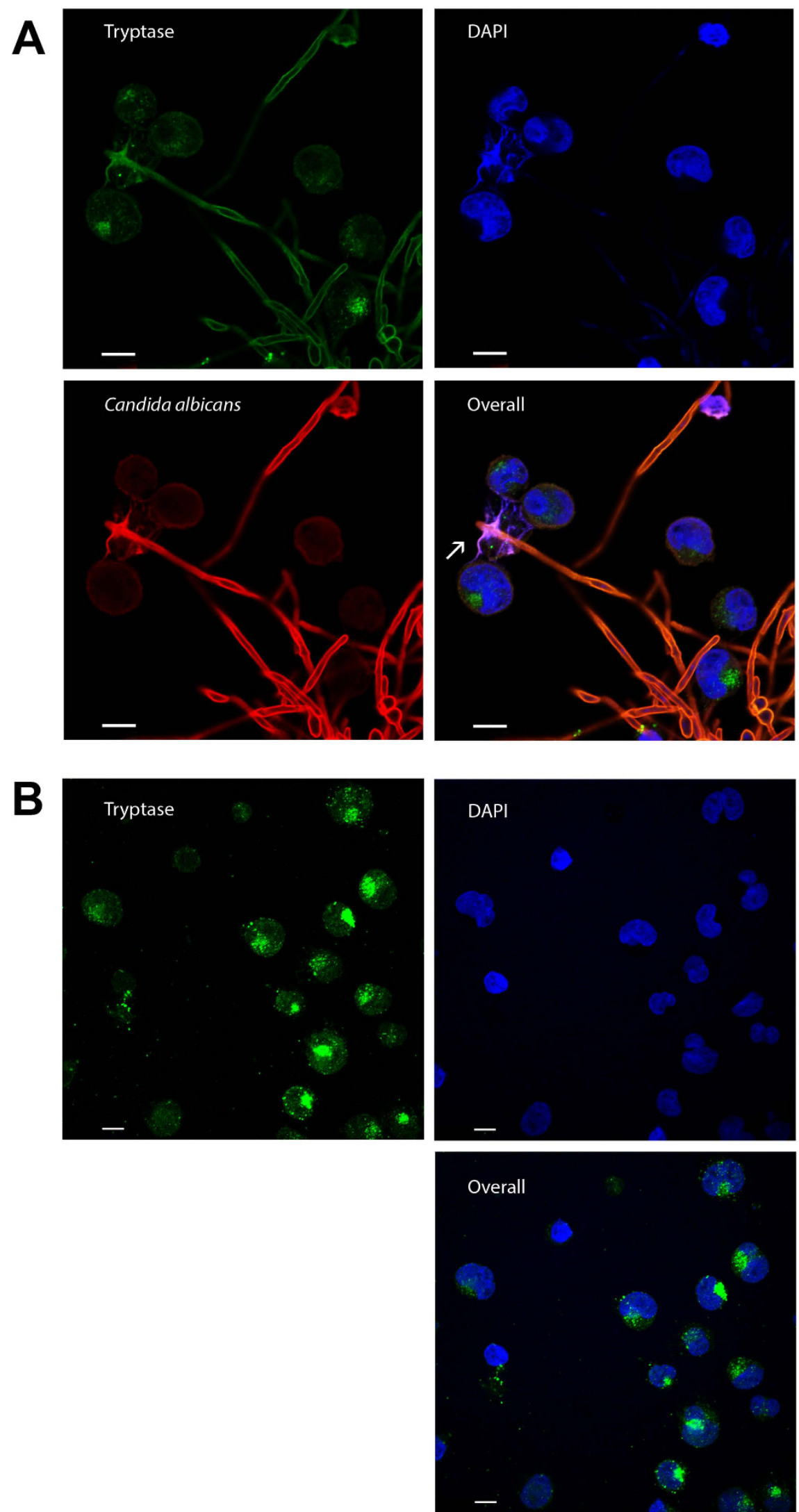

Figure 3. C. albicans induced MCETs in a time-dependent manner. Mast cells were infected for $6 \mathrm{~h}$ with C. albicans with an MOI 0.1 (A) or left uninfected (B). Shown are representative micrographs of indirect immunofluorescence from fixed and permeabilized samples with DNA (blue), mast cell tryptase (green) as well as C. albicans (red) stained samples. MCETs were identified by co-localization of extracellular laminar DNA with tryptase immunostaining (arrows). Scale bars, $10 \mu \mathrm{m}$. 
A

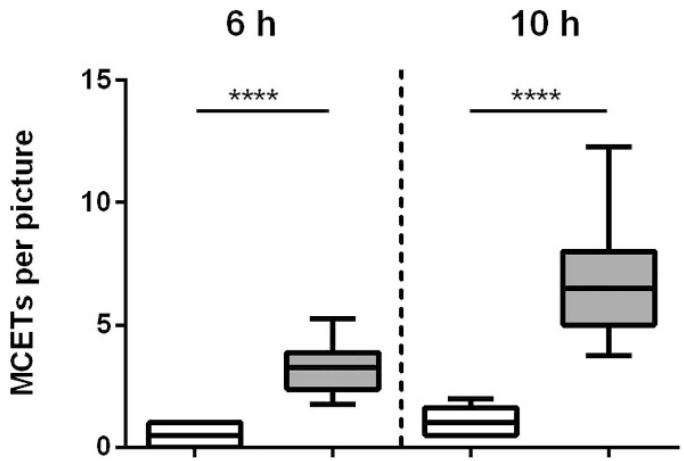

B
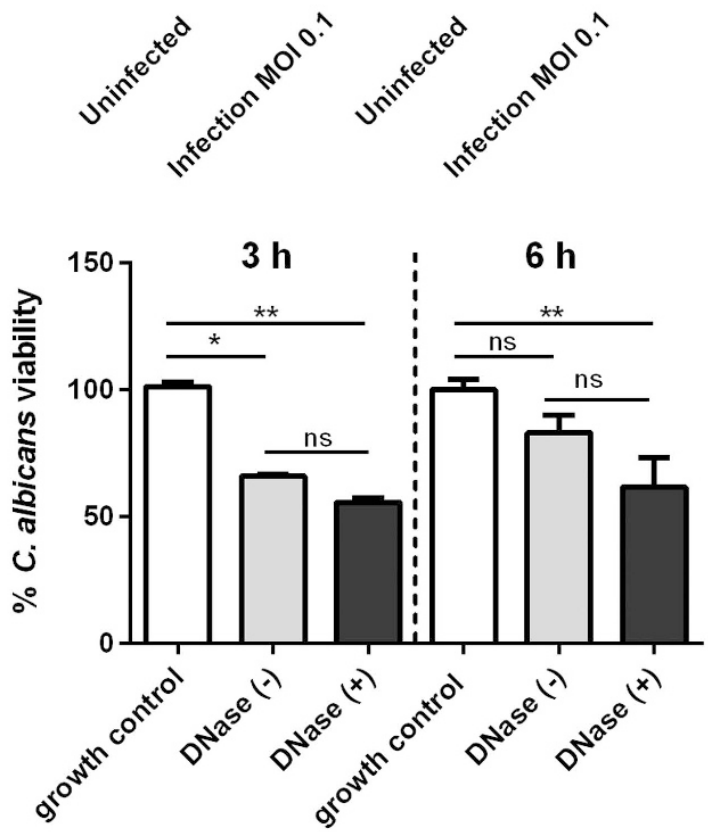

C

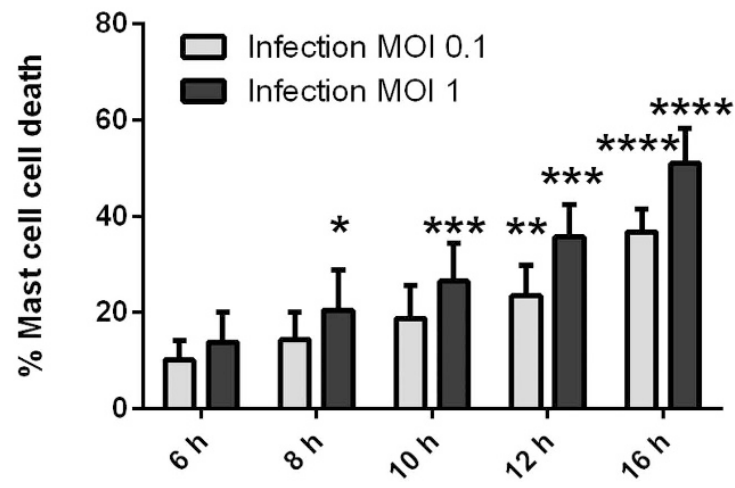

Figure 4. Quantification of cellular death and antifungal activity of mast cells. (A) The graph depicts the relative amount of MCETs per micrograph of C. albicans-infected mast cells (MOI 0.1) as compared to the uninfected mast cell control at two different time points. At both time points analysed (6h and $10 \mathrm{~h}$ ) the variation between MCETs compared to uninfected samples was analysed for statistical significance. (B) The graph represents the viability of fungal cells after normalization to the biological replicates $100 \%$ growth control. C. albicans viability is reduced in mast cell infection (MOI 1) up to $3 \mathrm{~h}$. (C) C. albicans-induced mast cell death in a time and dose-dependent manner as determined with Sytox green. The Y-axis represents the relative amount of dead cells after normalization to the mast cell lysis control. (A) Significance was analysed by t-test and by Tukey one-way ANOVA $(\mathbf{B}){ }^{*} \mathrm{P} \leq 0.05$. (B) Data are presented as means of at least six replicates (A) and $\mathrm{n}=3(6) \pm \mathrm{SD}$. (C) For cell death significance was analysed by Bonferroni two-way ANOVA ${ }^{*} \leq 0.05$ comparing to the mast cell uninfected control at each time point. Data represents $n=4(5) \pm S D$. 
C. albicans potently triggered mast cell death. To quantify mast cell death induced by C. albicans including MCET formation with a microscopy-independent method we used a microplate-based fluorescence assay with Sytox Green. The dye is impermeable for intact membranes staining DNA in the extracellular space and in dead cells. Cellular death was calculated as percentage of lysis control (Materials and Methods). Remarkably, mast cell infection with C. albicans resulted in $50 \%$ mast cell death after $10 \mathrm{~h}$ (Fig. 4C). Sytox-based cellular death quantification was confirmed by detection of lactate dehydrogenase (LDH) release into culture supernatants with similar results (data not shown). Similar levels of cell death were induced by C. albicans when cord blood-derived mast cells were infected (Fig. S3), confirming that our results are valid for primary immune cells.

This high percentage of cellular death cannot be explained by release of MCETs shown before, and therefore is governed by other mechanisms.

Internalized C. albicans can outgrow from mast cells. To assess other forms of mast cell death during interaction of mast cells and C. albicans we used live cell microscopy.

Interestingly, we found C. albicans yeast cells internalized into a few mast cells as soon as $30 \mathrm{~min}$ after initial infection. These yeast cells were able to grow intracellularly. Whether the fungal cells promote their own uptake or are actively phagocytized by mast cells remains to be determined. We observed intracellular propagation of $C$. albicans and subsequent germination resulted in collapse of mast cell plasma membranes. (Fig. 5A, arrow; Movie S2 and S3). Inside-out growth is a rare event estimated to occur in approximately $1 \%$ of all imaged cells.

We observed collapse of the membrane of the mast cells induced by extracellular C. albicans hyphae that grew towards the human cell (Fig. 5B, asterisk; Movie S3). Outside-in growth is a much more common event observed in around $15-20 \%$ of all imaged cell.

The live cell microscopy revealed a plausible explanations for the high mast cell death and putative outgrowth mechanism of C. albicans.

\section{Discussion}

Mast cells are crucial players in inflammatory and allergic processes. In recent years a growing body of evidence shed new light on a broader role of mast cells in host immunity ${ }^{6,24,34}$. Opportunistic fungal pathogens are an increasing burden to modern health care, due to a growing number of immunocompromised individuals as well as emergence of treatment-refractory strain $\mathrm{s}^{35}$. Commensal and ubiquitous opportunistic fungi colonize or enter the body via mast cell-rich tissues. A role of mast cells in antifungal immunity is therefore likely, however, virtually unexplored.

We hypothesized that $C$. albicans, one of the most frequent microbial pathogens and a common commensal on mucosal surfaces is recognized by human mast cells. Using an in vitro model we studied mast cells and C. albicans interactions using different approaches to qualitatively and quantitatively access this interplay. In this study we show that both primary- and cell line derived-mast cells responded to $C$. albicans in a specific and organized manner. The responses involved different stages, including initial, rapid degranulation followed by temporal release of cytokines that mediated cell recruitment and fungal recognition.

We demonstrated that mast cells initially affected C. albicans viability. The effect was temporary and decreased over a $6 \mathrm{~h}$ period, opposing the description of Trevisan et al. ${ }^{36}$ who showed that rat peritoneal mast cells permanently killed C. albicans. The same group additionally reported rat peritoneal mast cell degranulation towards unopsonized C. albicans ${ }^{36}$. Our study shows that mast cells degranulated more intensely towards opsonized C. albicans cells. Both, the previous and our study oppose a third using a rat-derived RBL-2H3 cell line ${ }^{37}$, in which the authors could not identify degranulation. Thus, we assume that these diverging results stem from differences in mast cell origin and maturation and from the fact that humans are natural hosts for C. albicans, whereas rats are not.

Human mast cells create an inflammatory environment by secreting a specific cytokine pattern during C. albicans challenge. Recruitment of innate immune cells, such as tissue macrophages and circulating neutrophils, is essential for clearing of fungal infection. We identified neutrophil-attracting chemokine IL-8 released by infected mast cells and translated the findings into a functional assay showing that human neutrophils migrate to infection supernatants. IL-8 release and the resulting recruitment of neutrophils is a known feature in mucosal tissues during candidiasis ${ }^{38}$, whereas the contribution of mast cells to this environment was not described. Importantly, IL-8 release was confirmed with primary mast cells, highlighting that the cytokine release was not restricted to the cell line HMC-1. The umbilical relation between mast cells and neutrophils has been demonstrated in an earlier report ${ }^{39}$ where injection of compound 48/80 in the mouse skin evoked an acute inflammatory reaction leading to a dose-dependent elevation of leukocyte numbers $4 \mathrm{~h}$ after challenge. Additional reports ${ }^{40,41}$ have also shown that tissue-resident mast cells control the early stage of neutrophil recruitment during tissue inflammation. In a 2015 report by Weber and colleagues ${ }^{42}$, mast cell deficiency inhibited neutrophil accumulation at the site of sensitization in a model of human allergic contact dermatitis.

In our experimental setting C. albicans-infected mast cells contribute to macrophage recruitment to a minor extent as supported by the secretion of inhibitory factor MIF, the diminished released of macrophage-chemoattractant MCP-1, and the absence of chemotattractant activity of the mast cell infection supernatants towards monocytes. The inflammatory response was changed at later time by 

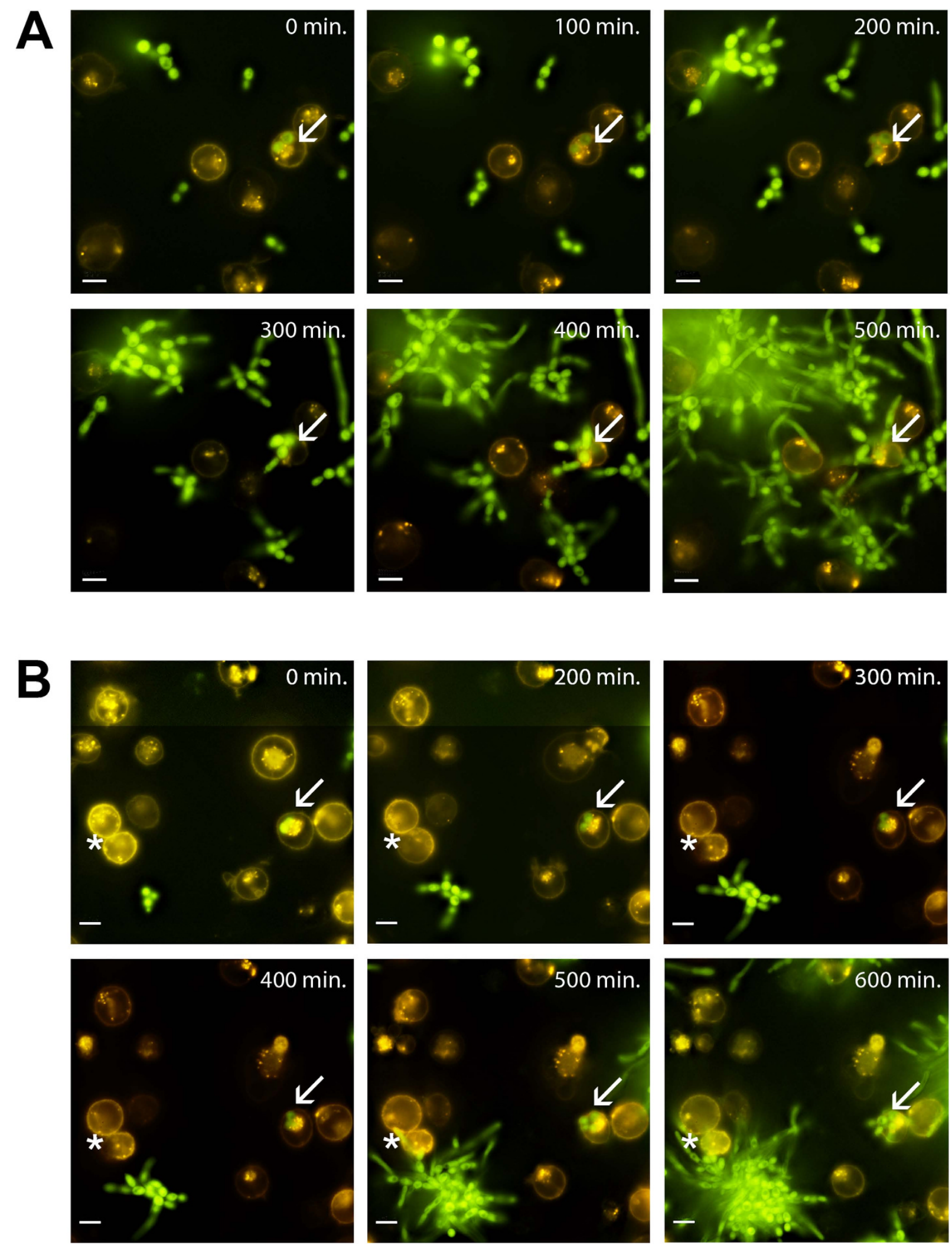

Figure 5. C. albicans internalization is followed by fungal outgrowth. Shown are images of the indicated time points for C. albicans infected mast cells (MOI 0.1). (A) Arrows show an intracellular yeast cell (GFPexpressing C. albicans strain CAI4 pENO1-GFP-CyC1t) replicating inside the mast cell (orange, membrane stain DiI) finally rupturing the plasma membrane as determined by loss of signal. An extracellular C. albicans hyphal tip growing towards a mast cell nudged the host cell and induced collapse of the plasma membrane $\left(^{*}\right)$. Complete movies are available as Movie S2 and S3 in the supplemental material. Scale bar, $10 \mu \mathrm{m}$. 
secretion of anti-inflammatory mediators and chemokines for adaptive immune cell recruitment. In an attempt to demonstrate chemotaxis of T-cells towards mast cell infection supernatants collected from late time points we observed high spontaneous migration of Jurkat cells. This is consistent with a previous report $^{43}$ and likely to mask T-cell migration towards specific chemoattractants present in our supernatants. In accordance to this notion, $\mathrm{CD} 4^{+} \mathrm{T}$-cell-mediated responses have been observed in models of fungal infection where antibiotic use caused C. albicans overgrowth and increased levels of mast cells proliferation $^{44}$.

Extracellular trap formation was first described in neutrophils by Brinkmann et al. ${ }^{45}$ and later in several other immune cells ${ }^{46-48}$. Extracellular traps act as danger signals in infection or inflammatory diseases, as extracellular release of proteases and other injurious cell constituents can exacerbate inflammatory processes ${ }^{33,49}$. In 2008 von Kockritz-Blickwede et al. ${ }^{6}$ showed that mast cells release chromatin decorated with granule proteins in extracellular filaments that bind to and kill bacteria. Interestingly, MCET release appears to be a mechanism of immune defence present in the mast cell toolbox against fungal pathogens as both primary cells and HMC-1 release MCETs upon C. albicans stimulation. In contrast to bacteria ${ }^{6,14}$, however, C. albicans viability was not affected by the mast cell-derived DNA fibbers and thus MCETs rather contribute to physical restriction of fungal pathogens.

Since MCET release alone was not sufficient to explain high mast cell death rates during infection, we decided to assess other forms of cellular death by live cell microscopy. We found that C. albicans could be internalized into mast cells, which seemed to occur rarely. The live cell imaging setup used does not unambiguously allow determining whether a Candida cell is within or attached to a mast cell.

Nevertheless, the growth of C. albicans hyphae towards and until attachment to mast cells from the outside (outside-in growth) resulted in loss of membrane dye and thus integrity of the host cell membrane, clearly demonstrating that mast cells are ruptured by C. albicans. This, at least in part, explains the high mast cell death during interaction with C. albicans, because it is a more frequent event (in 15-20\% of the cells). C. albicans can induce cell death in host cells. For instance, mucosal spread of C. albicans involves the adherence to and invasion of epithelial cells resulting in tissue damage $\mathrm{e}^{50}$. In macrophages, for instance, both yeast ${ }^{51}$ and hyphae ${ }^{52}$ can promote intracellular escape and host cell death. However, the exact mechanism and the in vivo relevance of $C$. albicans-induced mast cell death needs to be determined in further studies.

The described processes (Fig. 6) strongly suggest a role of mast cells as sentinels during the initial phase of fungal infection. Mast cells launched an immune response that contributes to elimination of the encountered fungal pathogen either directly by mast cell-dependent contact or indirectly by the recruitment of other immune cells. Nevertheless further investigation in vivo will help to access the contribution of mast cells to antifungal defence.

In spite of our findings that mast cells mounted a strong immune response against C. albicans, allergic reactions in humans are rarely triggered by this fungus alluding to a tolerance mechanism of the host ${ }^{53}$. Knowledge about this tolerance mechanism could help to improve treatment of common fungal-mediated asthma.

\section{Methods}

C. albicans culture conditions. C. albicans strains used in this study were: clinical isolate SC5314 (wild type) ${ }^{54}$ and for live cell imaging C. albicans (CAI4) ENO1 promoter GFP fusion.

For all experiments, fungal cultures were inoculated overnight in synthetic complete dropout medium $+2 \%$ glucose (SC) at $30^{\circ} \mathrm{C}$. A fresh subculture was inoculated in SC medium for $3 \mathrm{~h}$ at a starting cell number of $1 \times 10^{7}$ cells $/ \mathrm{ml}$ followed by $30 \mathrm{~min}$ opsonisation using pooled and heat-inactivated human serum $2 \%$ final concentration. C. albicans cells were centrifuged and re-suspended in PBS. The number of yeast cells was adjusted to the desired multiplicity of infection (MOI) prior to infection.

Plasmids and transformation of $C$. albicans. For this study, we generated a constitutively GFPexpressing C. albicans strain. A C. albicans-specific $\mathrm{GFP}^{55}$ (Genscript) was integrated into pUC57 via PstI and XbaI restriction sites for further integration into $\mathrm{pCaEXP}^{56}$. pCaEXP was linearized via StuI restriction digestion in the RP10 gene for integration of pENO1-GFP in strain CAI $4^{57}$, resulting in a $C$. albicans strain (CAI4 pENO1-GFP-CyC1t) constitutively expressing $\mathrm{GFP}^{58}$. Positive transformants were selected by plating on SC-uridine medium and the insertion of the GFP construct in the C. albicans genome was confirmed by sequencing.

Cell culture conditions. Cells from the human mast cell line HMC-1 ${ }^{59}$ and U937 cells (human monocytic cell line) were maintained in RPMI 1640-GlutaMax (Life Technologies) supplemented with $10 \%$ fetal calf serum, $100 \mathrm{U} / \mathrm{ml}$ penicillin and $100 \mu \mathrm{g} / \mathrm{ml}$ streptomycin (Lonza). Cord blood-derived mast cells (CBMCs) from CD34 ${ }^{+}$selected cells (Miltenyi Biotec) were cultivated in StemPro-34 SFM medium (Invitrogen) supplemented with $100 \mathrm{ng} / \mathrm{mL}$ recombinant human SCF (hSCF, Peprotech) and $10 \mathrm{ng} / \mathrm{mL}$ human IL-6 (Peprotech) (first week: additionally $10 \mathrm{ng} / \mathrm{ml}$ human IL-3) for 4 weeks and then maintained as previously described ${ }^{60}$. All cells were kept at $37^{\circ} \mathrm{C}, 5 \% \mathrm{CO}_{2}$. Medium was refreshed every 2-3 days. The culture was routinely checked for Mycoplasma contamination (MycoAlert, mycoplasma detection kit, Invitrogen). 


\section{Mast cell responses to $C$. albicans}

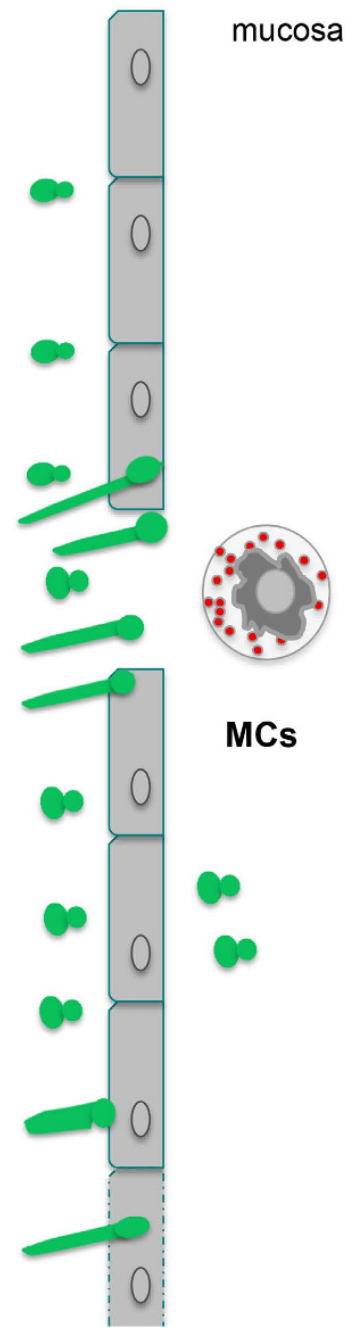

\section{Immediate response}

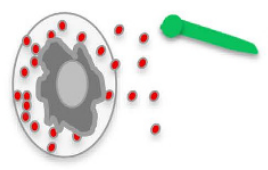

- Degranulation

- Reduced viability of $C$. albicans

\section{Intermediate response}

- Proinflammatory cytokines and neutrophil recruitment

- MCET release

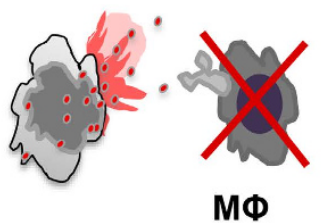

\section{Late} response

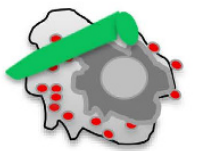

- C. albicans outgrowth from the mast cell

- Anti-inflammatory cytokines and recruitment of cells from adaptive immunity

Figure 6. Summary of mast cell and $C$. albicans interaction. Our findings indicate that mast cells specifically respond to $C$. albicans by degranulation, secretion of cytokines and chemokines, internalization of C. albicans and the release of MCETs. Interestingly, the processes seem to be organised and thus can be divided into three time periods: Initial (up to $3 \mathrm{~h}$ ), intermediate ( $3 \mathrm{~h}$ to $12 \mathrm{~h}$ ), and late responses $(>12 \mathrm{~h}$ ).

Prior to any assays, mast cells were primed with 25 nM PMA (12-myristate-13-acetate, Sigma-Aldrich) for $15 \mathrm{~min}$ at $3^{\circ} \mathrm{C}$ as previously described ${ }^{6}$. Cells were then centrifuged for $10 \mathrm{~min}$ at $300 \times \mathrm{g}$ and re-suspended in RPMI without fetal calf serum or antibiotics for the assays.

$\mathrm{N}$-acetyl- $\beta$-D-hexosaminidase release assay. $\quad \beta$-hexosaminidase secretion was measured according to a previous report ${ }^{61}$ with minor modifications. HMC- 1 cells $\left(1 \times 10^{5}\right.$ cells/well $)$ were infected, in a 96-well plate with different cell numbers of C. albicans (MOI 0.1, 1 and 10) or left untreated. After $1 \mathrm{~h}$ supernatants from technical replicates were pooled and added to new 96-well plates in triplicate.

Mast cell cytokine release. Mast cells $\left(1 \times 10^{6}\right.$ cells/well) were seeded in 24 -well plates (BD Falcon) and infected at $37^{\circ} \mathrm{C}, 5 \% \mathrm{CO}_{2}$ with $\mathrm{C}$. albicans at MOI $0.1,1$ or left untreated. C. albicans without any further additives was used as a fungal growth control. After infection, cells were centrifuged at $300 \times \mathrm{g}$ and supernatants collected and pooled. Debris in the mixture was further removed by centrifugation at $3000 \times \mathrm{g}$ for $10 \mathrm{~min}$ at $4^{\circ} \mathrm{C}$. The supernatants were harvested, shock-frozen in liquid nitrogen and stored at $-80^{\circ} \mathrm{C}$. The cytokine levels in the supernatants from the infections and respective controls were analysed using the Bio-Plex human cytokine 27-plex and 21-plex panel (Bio-Rad Inc., USA) for the following cytokines: IL-1 $\beta$, IL-1ra, IL-2, IL-4, IL-5, IL-6, IL-7, IL-8, IL-9, IL-10, IL-12, IL-13, IL-15, IL-16, IL-17, IL-18, IL-5, Eotaxin, FGF Basic, G-CSF, GM-CSF, IFN- $\gamma$, IP-10, MCP-1, MIP-1a, PDGF-BB, MIP-1 $\beta$, RANTES, TNF- $\alpha$, VEGF, IL-1 $\alpha$, IL-2RA, IL-12, CTACK, GRO $\alpha$, HGF, IFN- $\alpha 2$, LIF, 
MCP-3, M-CSF, MIF, MIG, $\beta$-NGF, SCF, SCGF- $\beta$, SDF- $1 \alpha$, TNF- $\beta$, TRAIL. Samples were mixed with antibody-coated beads that have a unique fluorescent intensity for the above cytokines. Anti-cytokine antibody PE-conjugated with streptavidin was added and the fluorescent signals were detected using a multiplex array reader Bio-Plex 200 System (Bio-Rad Laboratories). Raw data were initially measured as relative fluorescence intensities and then converted to cytokine concentrations based on the standard curve generated from reference concentrations supplied by the manufacturer.

Concentrations of IL-8 in supernatants of C. albicans-infected CBMC were measured using a human IL-8 enzyme-linked immunosorbent assay (ELISA) MAX kit (Biolegend, eBioscience USA). Primary cells were infected with $C$. albicans at MOI1 or left uninfected for $6 \mathrm{~h}$. Supernatants were harvested and stored as described above.

Chemotaxis assay for human neutrophils and monocytes. Neutrophils were harvested from blood of healthy volunteers according to the recommendations of the local ethical committee (Regionala etikprövningsnämnden i Umeå). Fully informed consent was obtained, and all investigations were conducted according to the principles stated in the Declaration of Helsinki. Neutrophils were isolated from venous blood as previously described ${ }^{32}$. Chemotactic migration of neutrophils and monocytes towards supernatants of mast cells infected with C. albicans (MOI 0.1), uninfected controls or the equivalent amount of fungal cells was measured using a transwell system as previously described ${ }^{32}$. Neutrophils migration was accessed for $30 \mathrm{~min}$ and for monocytes migration was accessed for $90 \mathrm{~min}$. Mast cell infected supernatants tested were collected and stored as described for the cytokine release assay at $6 \mathrm{~h}$, $12 \mathrm{~h}$ and $16 \mathrm{~h}$.

Immunostaining and microscopic analysis of mast cells. Mast cells $\left(1 \times 10^{5}\right.$ cells/well $)$ were seeded onto cover slips coated with $1 \%$ poly-l-lysine (Sigma-Aldrich) in 24-well plates and infected with C. albicans (MOI 0.1, 1). Uninfected mast cells were used as control. After $6 \mathrm{~h}$ cells were fixed using $2 \%$ paraformaldehyde and stored at $4^{\circ} \mathrm{C}$.

For visualization of MCETs, primary antibodies directed against human mast cell tryptase (clonal AA1, mouse, DAKO) and C. albicans antibody (mouse monoclonal, ProSci) diluted in blocking solution were applied overnight at $4^{\circ} \mathrm{C}$. Primary antibodies were detected with Alexa Fluor 488- and 568-conjugated secondary antibodies (Life Technologies). DNA was visualized with DAPI (4', 6'-diamidino-2-phenylindole; Life Technologies). Specimens were mounted in Pro-Long Diamond (Life Technologies).

Imaging data were acquired using a fully motorized inverted microscope (Nikon A1R Laser Scanning Confocal Microscope) with $60 \times$ oil immersion lens (Plan Apochromat VC; Nikon, Tokyo, Japan) under control of the NIS-Elements microscope imaging software (Nikon). Final image composition was done using Adobe Photoshop CS5 (San Jose, CA).

For live cell microscopy mast cells $\left(2 \times 10^{5}\right.$ cells/well $)$ were stained with Vybrant DiI Cell-labelling solution (Invitrogen) according to the manufacturer's instructions and seeded into a 35-mm glass-bottom micro- well dish (MatTek, Ashland, MA, USA). Mast cells were infected with C. albicans strain (CAI4 pENO1-GFP-CyC1t) at MOI 1 and kept at cell-culture conditions throughout the measurement. Frames were captured $30 \mathrm{~min}$ post-infection at $60 \times$ magnification every $10 \mathrm{~min}$ for a period of $16 \mathrm{~h}$ using the previously described microscope.

Microscopic quantification was performed using DAPI immuno-stained image samples from six biological replicates. Images analysed had $130 \pm 30$ cells per picture and for each infection condition a total of at least 1000 cells were analysed. The total number of cells was determined by ImageJ version 2.0. The number of cells that underwent MCET formation was scored from binary images in a blinded fashion by two trained researchers. Final scores were defined as MCETs per field of view and plotted by condition and infection end-point.

From 10 independent live cell movies the \% of cells undergoing inside-out growth was determined as the ratio of [(inside-out growth)/100\% total cells)]. Similar the $\%$ of cells undergoing outside-in growth was determine as the ratio of [(outside-in side growth)/100\% total cells)]. In both cases a total of 80 cells was analysed.

Fungal viability measurement. To determine the antifungal effect of mast cells we compared fungal viability in the presence of mast cells as follows: Mast cells $\left(5 \times 10^{4}\right.$ cells/well $)$ were infected with C. albicans at MOI 1 for 3 and $6 \mathrm{~h}$ in a 96-well plate coated with poly-l-lysine. The same amount of $C$. albicans served as $100 \%$ control. To test for contribution of MCETs to fungal viability we added DNaseI (Sigma Aldrich) prior to infection to one set of experiments. At the end point of the experiment, DNaseI and subsequently Triton-X100 to a final concentration of $10 \%$ were added to all wells. The medium was removed and fungal viability (ATP) was determined using CellTiter-Glo cell viability kit (Promega) in a luminometer (Tecan Infinite F200) as previously described ${ }^{62}$.

To normalize all values for comparable ATP signals, values were multiplied by the factor: [average of technical replicates of $100 \%$ growth control]/[average of biological replicates of $100 \%$ growth controls]. Using these normalized values, the antifungal effect was determined as the ratio of [(infected MCsuninfected MCs) $/ 100 \%$ growth C. albicans)].

To assure that any differences in cell viability were not due to loss of cells during washing we measured absorbance before triton lysis and after adding CellTiter-Glo reagent and found no notable variation. 
Cell death assay. Cellular death of mast cells was quantified using a Sytox Green-based assay as previously described for human neutrophils ${ }^{32}$. Mast cells $\left(5 \times 10^{4}\right.$ cells/well) were seeded in a black 96-well plate in the presence of $2.5 \mu \mathrm{M}$ Sytox Green and infected with C. albicans - MOI 0.1, 1- or left untreated. The same amount of $C$. albicans served as viability control whereas triton-lysed mast cells served as a reference for $100 \%$ cellular death.

Statistics. Statistical analysis was performed using GraphPad Prism Software 6.01 (GraphPad Software, La Jolla, CA, USA).

Cytokine profile $n=3$ (3), $\beta$-hexosaminidase $n=4$ (4), chemotaxis $n=5$ (3) and fungal viability $\mathrm{n}=3$ (6) results were analysed applying one-way ANOVA with Tukey's post-test. ELISA results $n=2$ (3), 30 min end-point chemotaxis quantification and microscopic quantification of MCETs $n=6$ were analysed using t-test with Welsh correction to compare uninfected control and infection conditions in the corresponding time point. Cell death were analysed as $n=4$ (5) experiments applying two-way ANOVA with Bonferroni's post-test.

For all analyses $\mathrm{p}$-values $<0.05$ was considered statistically significant.

\section{References}

1. Mikulska, M., Del Bono, V., Ratto, S. \& Viscoli, C. Occurrence, presentation and treatment of candidemia. Expert Rev Clin Immunol 8, 755-765, doi: 10.1586/eci.12.52 (2012).

2. Patterson, T. F. Advances and challenges in management of invasive mycoses. Lancet 366, 1013-1025, doi: 10.1016/S01406736(05)67381-3 (2005).

3. Montravers, P. et al. A multicentre study of antifungal strategies and outcome of Candida spp. peritonitis in intensive-care units. Clin Microbiol Infect 17, 1061-1067, doi: 10.1111/j.1469-0691.2010.03360.x (2011).

4. Bassetti, M. et al. Epidemiology, species distribution, antifungal susceptibility, and outcome of candidemia across five sites in Italy and Spain. J Clin Microbiol 51, 4167-4172, doi: 10.1128/JCM.01998-13 (2013).

5. Abraham, S. N. \& St John, A. L. Mast cell-orchestrated immunity to pathogens. Nat Rev Immunol 10, 440-452, doi: 10.1038/ nri2782 (2010).

6. von Kockritz-Blickwede, M. et al. Phagocytosis-independent antimicrobial activity of mast cells by means of extracellular trap formation. Blood 111, 3070-3080, doi: 10.1182/blood-2007-07-104018 (2008).

7. Cruse, G. et al. Human lung mast cells mediate pneumococcal cell death in response to activation by pneumolysin. J Immunol 184, 7108-7115, doi: 10.4049/jimmunol.0900802 (2010).

8. Rodriguez, A. R. et al. Mast cell TLR2 signaling is crucial for effective killing of Francisella tularensis. J Immunol 188, 5604-5611, doi: 10.4049/jimmunol.1200039 (2012).

9. Selander, C., Engblom, C., Nilsson, G., Scheynius, A. \& Andersson, C. L. TLR2/MyD88-dependent and -independent activation of mast cell IgE responses by the skin commensal yeast Malassezia sympodialis. J Immunol 182, 4208-4216, doi: 10.4049/ jimmunol.0800885 (2009).

10. Urb, M., Pouliot, P., Gravelat, F. N., Olivier, M. \& Sheppard, D. C. Aspergillus fumigatus induces immunoglobulin E-independent mast cell degranulation. J Infect Dis 200, 464-472, doi: 10.1086/600070 (2009).

11. Saluja, R., Metz, M. \& Maurer, M. Role and relevance of mast cells in fungal infections. Front Immunol 3, 146, doi: 10.3389/ fimmu.2012.00146 (2012).

12. Urb, M. \& Sheppard, D. C. The role of mast cells in the defence against pathogens. PLoS Pathog 8, e1002619, doi: 10.1371/journal. ppat.1002619 (2012).

13. Supajatura, V. et al. Differential responses of mast cell Toll-like receptors 2 and 4 in allergy and innate immunity. $J$ Clin Invest 109, 1351-1359, doi: 10.1172/JCI14704 (2002).

14. Scheb-Wetzel, M., Rohde, M., Bravo, A. \& Goldmann, O. New Insights into the Antimicrobial Effect of Mast Cells against Enterococcus faecalis. Infect Immun 82, 4496-4507, doi: 10.1128/IAI.02114-14 (2014).

15. Carlos, D. et al. TLR2-dependent mast cell activation contributes to the control of Mycobacterium tuberculosis infection. Microbes Infect 11, 770-778, doi: 10.1016/j.micinf.2009.04.025 (2009).

16. Inoue, M. \& Shinohara, M. L. Clustering of pattern recognition receptors for fungal detection. PLoS Pathog 10, e1003873, doi: 10.1371/journal.ppat.1003873 (2014).

17. Shoham, S., Huang, C., Chen, J. M., Golenbock, D. T. \& Levitz, S. M. Toll-like receptor 4 mediates intracellular signaling without TNF-alpha release in response to Cryptococcus neoformans polysaccharide capsule. J Immunol 166, 4620-4626 (2001).

18. Kimura, Y. et al. Dectin-1-mediated signaling leads to characteristic gene expressions and cytokine secretion via spleen tyrosine kinase (Syk) in rat mast cells. J Biol Chem 289, 31565-31575, doi: 10.1074/jbc.M114.581322 (2014).

19. Olynych, T. J., Jakeman, D. L. \& Marshall, J. S. Fungal zymosan induces leukotriene production by human mast cells through a dectin-1-dependent mechanism. J Allergy Clin Immunol 118, 837-843, doi: 10.1016/j.jaci.2006.06.008 (2006).

20. Yang, Z. \& Marshall, J. S. Zymosan treatment of mouse mast cells enhances dectin-1 expression and induces dectin-1-dependent reactive oxygen species (ROS) generation. Immunobiology 214, 321-330, doi: 10.1016/j.imbio.2008.09.002 (2009).

21. Wernersson, S. \& Pejler, G. Mast cell secretory granules: armed for battle. Nat Rev Immunol 14, 478-494, doi: 10.1038/nri3690 (2014).

22. Maurer, M. et al. Skin mast cells control T cell-dependent host defense in Leishmania major infections. FASEB J 20, 2460-2467, doi: 10.1096/fj.06-5860 com (2006).

23. Echtenacher, B., Mannel, D. N. \& Hultner, L. Critical protective role of mast cells in a model of acute septic peritonitis. Nature 381, 75-77, doi: 10.1038/381075a0 (1996).

24. Fukuishi, N. et al. Does beta-Hexosaminidase Function Only as a Degranulation Indicator in Mast Cells? The Primary Role of beta-Hexosaminidase in Mast Cell Granules. J Immunol 193, 1886-1894, doi: 10.4049/jimmunol.1302520 (2014).

25. Caughey, G. H. Mast cell tryptases and chymases in inflammation and host defense. Immunol Rev 217, 141-154, doi: 10.1111/j.1600-065X.2007.00509.x (2007)

26. Oh, S. W. et al. Tryptase inhibition blocks airway inflammation in a mouse asthma model. J Immunol 168, 1992-2000 (2002).

27. Marshall, J. S. Mast-cell responses to pathogens. Nat Rev Immunol 4, 787-799, doi: 10.1038/nri1460 (2004).

28. Shin, K. et al. Mouse mast cell tryptase mMCP-6 is a critical link between adaptive and innate immunity in the chronic phase of Trichinella spiralis infection. J Immunol 180, 4885-4891 (2008).

29. Thakurdas, S. M. et al. The mast cell-restricted tryptase mMCP-6 has a critical immunoprotective role in bacterial infections. $J$ Biol Chem 282, 20809-20815, doi: 10.1074/jbc.M611842200 (2007). 
30. Wang, B. et al. Cutting edge: Deficiency of macrophage migration inhibitory factor impairs murine airway allergic responses. $J$ Immunol 177, 5779-5784 (2006).

31. Rumsaeng, V. et al. Human mast cells produce the CD4+ T lymphocyte chemoattractant factor, IL-16. J Immunol 159, 29042910 (1997).

32. Hosseinzadeh, A., Messer, P. K. \& Urban, C. F. Stable Redox-Cycling Nitroxide Tempol Inhibits NET Formation. Front Immunol 3, 391, doi: 10.3389/fimmu.2012.00391 (2012).

33. Lin, A. M. et al. Mast cells and neutrophils release IL-17 through extracellular trap formation in psoriasis. J Immunol 187, 490-500, doi: 10.4049/jimmunol.1100123 (2011).

34. Scheb-Wetzel, M., Rohde, M., Bravo, A. \& Goldmann, O. New insights into the antimicrobial effect of mast cells against Enterococcus faecalis. Infect Immun. doi: 10.1128/IAI.02114-14 (2014).

35. Guery, B. P. et al. Management of invasive candidiasis and candidemia in adult non-neutropenic intensive care unit patients: Part I. Epidemiology and diagnosis. Intensive Care Med 35, 55-62, doi: 10.1007/s00134-008-1338-7 (2009).

36. Trevisan, E. et al. Mast Cells Kill Candida albicans in the Extracellular Environment but Spare Ingested Fungi from Death. Inflammation. doi: 10.1007/s10753-014-9951-9 (2014).

37. Sakurai, A., Yamaguchi, N. \& Sonoyama, K. Cell Wall Polysaccharides of Candida albicans Induce Mast Cell Degranulation in the Gut. Biosci Microbiota Food Health 31, 67-70, doi: 10.12938/bmfh.31.67 (2012).

38. Dongari-Bagtzoglou, A. \& Kashleva, H. Candida albicans triggers interleukin-8 secretion by oral epithelial cells. Microb Pathog 34, 169-177 (2003).

39. Schramm, R., Schaefer, T., Menger, M. D. \& Thorlacius, H. Acute mast cell-dependent neutrophil recruitment in the skin is mediated by KC and LFA-1: inhibitory mechanisms of dexamethasone. J Leukoc Biol 72, 1122-1132 (2002).

40. De Filippo, K. et al. Mast cell and macrophage chemokines CXCL1/CXCL2 control the early stage of neutrophil recruitment during tissue inflammation. Blood 121, 4930-4937, doi: 10.1182/blood-2013-02-486217 (2013).

41. Pimentel, T. A., Sampaio, A. L., D’Acquisto, F., Perretti, M. \& Oliani, S. M. An essential role for mast cells as modulators of neutrophils influx in collagen-induced arthritis in the mouse. Lab Invest 91, 33-42, doi: 10.1038/labinvest.2010.140 (2011).

42. Weber, F. C. et al. Neutrophils are required for both the sensitization and elicitation phase of contact hypersensitivity. J Exp Med 212, 15-22, doi: 10.1084/jem.20130062 (2015).

43. Butler, J. T., Samantaray, S., Beeson, C. C., Ray, S. K. \& Banik, N. L. Involvement of calpain in the process of Jurkat T cell chemotaxis. J Neurosci Res 87, 626-635, doi: 10.1002/jnr.21882 (2009).

44. Noverr, M. C., Noggle, R. M., Toews, G. B. \& Huffnagle, G. B. Role of antibiotics and fungal microbiota in driving pulmonary allergic responses. Infect Immun 72, 4996-5003, doi: 10.1128/IAI.72.9.4996-5003.2004 (2004).

45. Brinkmann, V. et al. Neutrophil extracellular traps kill bacteria. Science 303, 1532-1535, doi: 10.1126/science.1092385 (2004).

46. Simon, D. et al. Eosinophil extracellular DNA traps in skin diseases. J Allergy Clin Immunol 127, 194-199, doi: 10.1016/j. jaci.2010.11.002 (2011)

47. Mohanan, S., Horibata, S., McElwee, J. L., Dannenberg, A. J. \& Coonrod, S. A. Identification of macrophage extracellular trap-like structures in mammary gland adipose tissue: a preliminary study. Front Immunol 4, 67, doi: 10.3389/fimmu.2013.00067 (2013).

48. Morshed, M. et al. NADPH Oxidase-Independent Formation of Extracellular DNA Traps by Basophils. J Immuno. doi: 10.4049/ jimmunol.1303418 (2014).

49. Moretti, S. et al. The contribution of PARs to inflammation and immunity to fungi. Mucosal Immunol 1, 156-168, doi: 10.1038/ mi.2007.13 (2008).

50. Zhu, W. \& Filler, S. G. Interactions of Candida albicans with epithelial cells. Cell Microbiol 12, 273-282, doi: 10.1111/j.14625822.2009.01412.x (2010).

51. O'Meara, T. R. et al. Global analysis of fungal morphology exposes mechanisms of host cell escape. Nat Commun 6, 6741, doi: 10.1038/ncomms7741 (2015).

52. Uwamahoro, N. et al. The pathogen Candida albicans hijacks pyroptosis for escape from macrophages. MBio 5, e00003-00014, doi: 10.1128/mBio.00003-14 (2014).

53. McAlpine, S. M., Enoksson, M., Lunderius-Andersson, C. \& Nilsson, G. The effect of bacterial, viral and fungal infection on mast cell reactivity in the allergic setting. J Innate Immun 3, 120-130, doi: 10.1159/000323350 (2011).

54. Gillum, A. M., Tsay, E. Y. \& Kirsch, D. R. Isolation of the Candida albicans gene for orotidine-5'-phosphate decarboxylase by complementation of S. cerevisiae ura3 and E. coli pyrF mutations. Mol Gen Genet 198, 179-182 (1984).

55. Gerami-Nejad, M., Dulmage, K. \& Berman, J. Additional cassettes for epitope and fluorescent fusion proteins in Candida albicans. Yeast 26, 399-406, doi: 10.1002/yea.1674 (2009).

56. Care, R. S., Trevethick, J., Binley, K. M. \& Sudbery, P. E. The MET3 promoter: a new tool for Candida albicans molecular genetics. Mol Microbiol 34, 792-798 (1999).

57. Fonzi, W. A. \& Irwin, M. Y. Isogenic strain construction and gene mapping in Candida albicans. Genetics 134, 717-728 (1993).

58. Staab, J. F., Bahn, Y. S. \& Sundstrom, P. Integrative, multifunctional plasmids for hypha-specific or constitutive expression of green fluorescent protein in Candida albicans. Microbiology 149, 2977-2986 (2003).

59. Butterfield, J. H., Weiler, D., Dewald, G. \& Gleich, G. J. Establishment of an immature mast cell line from a patient with mast cell leukemia. Leuk Res 12, 345-355 (1988).

60. Xiang, Z., Moller, C. \& Nilsson, G. IgE-receptor activation induces survival and Bfl-1 expression in human mast cells but not basophils. Allergy 61, 1040-1046, doi: 10.1111/j.1398-9995.2006.01024.x (2006).

61. Ekoff, M., Moller, C., Xiang, Z. \& Nilsson, G. Coaggregation of FcepsilonRI with FcgammaRIIB Inhibits Degranulation but Not Induction of Bcl-2 Family Members A1 and Bim in Mast Cells. Allergy Asthma Clin Immunol 2, 87-97, doi: 10.1186/1710-14922-3-87 (2006).

62. Stylianou, M. et al. Antifungal application of nonantifungal drugs. Antimicrob Agents Chemother 58, 1055-1062, doi: 10.1128/ AAC.01087-13 (2014).

\section{Acknowledgments}

This study was funded by grants to CFU from the Swedish Research Council VR-M (K201299X-21961-01-3), the Åke Wiberg Foundation (3772734 and M14-0076) the Laboratory for Molecular Medicine Sweden (MIMS) and the Medical Faculty Umeå (316-886-10). JPL acknowledges travel support from the Swedish Foundation for Strategic Research. We would like to thank Ava Hosseinzadeh, Sandra Holmberg, Constantina Georgiadou, Maria Johanna Niemiec for their support and Nelson Gekara as well as Marc Röhm for critically reading the manuscript. Additionally, we would like to acknowledge Maria Ekoff and Irene Martinez Carrasco (Biochemical Imaging Centre - Umeå University) for technical support. 


\section{Author Contributions}

J.P.L., M.S., C.F.U wrote the main manuscript text and prepared all figures; J.P.L., M.S. conducted the experiments; G.N., contributed reagents/materials; J.P.L., M.S., C.F.U. analyzed the data and revised the manuscript. C.F.U. designed the study and conceived the experiments.

\section{Additional Information}

Supplementary information accompanies this paper at http://www.nature.com/srep

Competing financial interests: The authors declare no competing financial interests.

How to cite this article: Lopes, J.P. et al. Opportunistic pathogen Candida albicans elicits a temporal response in primary human mast cells. Sci. Rep. 5, 12287; doi: 10.1038/srep12287 (2015).

(c) (i) This work is licensed under a Creative Commons Attribution 4.0 International License. The images or other third party material in this article are included in the article's Creative Commons license, unless indicated otherwise in the credit line; if the material is not included under the Creative Commons license, users will need to obtain permission from the license holder to reproduce the material. To view a copy of this license, visit http://creativecommons.org/licenses/by/4.0/ 